\title{
Front Matter: Volume 11359
}

, "Front Matter: Volume 11359," Proc. SPIE 11359, Biomedical Spectroscopy, Microscopy, and Imaging, 1135901 (16 April 2020); doi: 10.1117/12.2571778

SPIE. Event: SPIE Photonics Europe, 2020, Online Only 


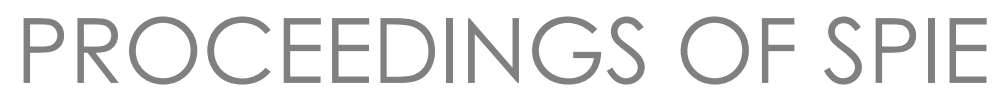

\title{
Biomedical Spectroscopy, Microscopy, and Imaging
}

\author{
Jürgen Popp \\ Csilla Gergely \\ Editors
}

6-10 April 2020

Online Only, France

Sponsored by

SPIE

Cosponsored by

City of Strasbourg (France)

Eurometropole (France)

CNRS (France)

Région Grand Est (France)

iCube (France)

Université de Strasbourg (France)

Cooperating Organisations

Photonics 21 (Germany)

EOS-European Optical Society (Germany)

Photonics Public Private Partnership (Belgium)

Photonics France (France)

Published by

SPIE

Volume 11359 
The papers in this volume were part of the technical conference cited on the cover and title page. Papers were selected and subject to review by the editors and conference program committee. Some conference presentations may not be available for publication. Additional papers and presentation recordings may be available online in the SPIE Digital Library at SPIEDigitalLibrary.org.

The papers reflect the work and thoughts of the authors and are published herein as submitted. The publisher is not responsible for the validity of the information or for any outcomes resulting from reliance thereon.

Please use the following format to cite material from these proceedings:

Author(s), "Title of Paper," in Biomedical Spectroscopy, Microscopy, and Imaging, edited by Jürgen Popp, Csilla Gergely, Proceedings of SPIE Vol. 11359 (SPIE, Bellingham, WA, 2020) Seven-digit Article CID Number.

ISSN: 0277-786X

ISSN: 1996-756X (electronic)

ISBN: 9781510634909

ISBN: 9781510634916 (electronic)

Published by

SPIE

P.O. Box 10, Bellingham, Washington 98227-0010 USA

Telephone +1 3606763290 (Pacific Time) · Fax +1 3606471445

SPIE.org

Copyright (C) 2020, Society of Photo-Optical Instrumentation Engineers.

Copying of material in this book for internal or personal use, or for the internal or personal use of specific clients, beyond the fair use provisions granted by the U.S. Copyright Law is authorized by SPIE subject to payment of copying fees. The Transactional Reporting Service base fee for this volume is $\$ 21.00$ per article (or portion thereof), which should be paid directly to the Copyright Clearance Center (CCC), 222 Rosewood Drive, Danvers, MA 01923. Payment may also be made electronically through CCC Online at copyright.com. Other copying for republication, resale, advertising or promotion, or any form of systematic or multiple reproduction of any material in this book is prohibited except with permission in writing from the publisher. The CCC fee code is 0277$786 \mathrm{X} / 20 / \$ 21.00$.

Printed in the United States of America by Curran Associates, Inc., under license from SPIE.

Publication of record for individual papers is online in the SPIE Digital Library.

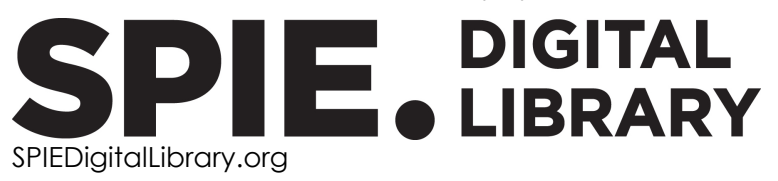

Paper Numbering: Proceedings of SPIE follow an e-First publication model. A unique citation identifier (CID) number is assigned to each article at the time of publication. Utilization of CIDs allows articles to be fully citable as soon as they are published online, and connects the same identifier to all online and print versions of the publication. SPIE uses a seven-digit CID article numbering system structured as follows:

- The first five digits correspond to the SPIE volume number.

- The last two digits indicate publication order within the volume using a Base 36 numbering system employing both numerals and letters. These two-number sets start with 00, 01, 02, 03, 04, 05, 06, 07, 08, 09, OA, OB ... 0Z, followed by 10-1Z, 20-2Z, etc. The CID Number appears on each page of the manuscript. 


\title{
Contents
}

\author{
vii Authors \\ ix Conference Committee
}

\section{RAMAN SPECTROSCOPY IMAGING I}

1135902 Prognosis, diagnosis and the influence of inhibitors: Raman spectroscopic study of radioresistant brain cancer stem-like cells (Invited Paper) [11359-1]

1135904 Potential and challenges of pathogen detection using Raman spectroscopy [11359-3]

RAMAN SPECTROSCOPY IMAGING II

1135907 Coherent anti-Stokes Raman scattering microscopy through a multimode fiber endoscope [11359-6]

1135908 Corneal collagen morpho-mechanics characterized by correlative optical microscopies (Invited Paper) [1 1359-7]

\section{IR SPECTROSCOPY AND IMAGING}

1135909 Modelling the wavelength-dependent response of SHG excitation efficiency (Invited Paper) [11359-8]

11359 OA Signal enhancement in microstructured silicon attenuated total reflection elements with quantum cascade laser-based spectroscopy [11359-9]

11359 OB Mid-infrared multispectral lensless imaging for wide-field and label-free microbial identification [11359-10]

11359 OC Does chaotic scattering affect the extinction efficiency in quasi-spherical scatterers? [11359-11]

\section{OPTICAL COHERENCE TOMOGRAPHY I}

$11359 \mathrm{OE} \quad$ Compressional optical coherence elastography as a tool for feasible in vivo histology-like morphological segmentation of cancer-tissue constituents (Invited Paper) [11359-13]

11359 OF Line-field confocal optical coherence tomography: technology and application in dermatology [11359-14] 
11359 OG Optical coherence elastography for characterization of natural interstitial gaps and laserirradiation-produced porosity in corneal and cartilaginous samples [11359-15]

$11359 \mathrm{OH} \quad$ Characterization of elastic nonlinear properties of the tissues using compressional optical coherence elastography [11359-16]

\section{OPTICAL COHERENCE TOMOGRAPHY II}

11359 OK Imaging photothermal-induced expansion of bone during laser osteotomy by phase-sensitive OCT: preliminary results [11359-19]

\section{OPTICAL COHERENCE TOMOGRAPHY III}

11359 OP Methods for reducing relative intensity noise in swept-source mid-infrared OCT [11359-24]

11359 OS New algorithms to characterize and classify ophthalmic images (Invited Paper) [11359-27]

\section{ADVANCED SPECTROSCOPY AND IMAGING I}

11359 OT Advanced biomedical multiphoton fluorescence microscopy with a large band excitation system (Invited Paper) [11359-33]

11359 OU Study of calcium signaling dynamics in single platelets using optical activation methods [11359-34]

11359 OV Phototoxic effects of a genetically-encoded photosensitizer minisOG in tumor spheroids induced by continuous wave or pulsed laser irradiation [1 1359-35]

\section{ADVANCED SPECTROSCOPY AND IMAGING II}

1135910 Tight focal spots using azimuthally polarised light from a Fresnel cone [1 1359-40]

\section{ADVANCED SPECTROSCOPY AND IMAGING IV}

11359 1C Convolutional autoencoder-based reconstruction of vascular structures in photoacoustic images [11359-47]

iv 
11359 1D Laser speckle time-series correlation analysis for bacteria activity detection [1 1359-48]

11359 1E A combination of imaging techniques for dental medicine: from x-rays radiography and 3D CBCT to OCT [1 1359-49]

$11359 \mathrm{IF} \quad$ Calibration routine for incoherent optical fiber bundles for medical ultra-high spatial resolution fiber spectroscopy [11359-50]

$113591 \mathrm{H} \quad$ Semi-analytical full-wave model of OCT-scan formation for various degrees of OCT-beam focusing with implication of motion of scatterers [1 1359-53]

$113591 \mathrm{~J} \quad$ Infrared quantum cascade laser spectroscopy as non-invasive diagnostic tests for human diseases [11359-55]

11359 1N A new macro-imager based on Tpx3Cam optical camera for PLIM applications [11359-60]

1135910 Quantitative assessment of the breast tumor stroma using cross-polarization OCT and multiphoton tomography [11359-61]

11359 IP Characterization of auto-fluorescence urine crystals from gout patients using confocal microscopy and micro-Raman system for urolithiasis prediction [1 1359-62]

11359 IR New approach to the study of cell cytotoxicity using high-resolution coherence phaseinterference microscopy [11359-64]

11359 1T Raman-based high-resolution detection of ${ }^{13} \mathbf{C O}_{2}$ isotopes in human breath [1 1359-66]

$113591 \mathrm{U} \quad$ Monitoring of blood flow by speckle correlation sensor [1 1359-67]

11359 1W Keratoconus apex positions impact on visual acuity and contrast sensitivity [11359-70] 
Proc. of SPIE Vol. 11359 1135901-6

Downloaded From: https://www.spiedigitallibrary.org/conference-proceedings-of-spie on 26 Apr 2023
Terms of Use: https://www.spiedigitallibrary.org/terms-of-use 


\section{Authors}

Numbers in the index correspond to the last two digits of the seven-digit citation identifier (CID) article numbering system used in Proceedings of SPIE. The first five digits reflect the volume number. Base 36 numbering is employed for the last two digits and indicates the order of articles within the volume. Numbers start with 00, 01, 02, 03, 04, 05, 06, 07, 08, 09, 0A, 0B...0Z, followed by 10-12, 20-2Z, etc.

\begin{tabular}{|c|c|}
\hline Akimov, Denis, 07 & Haq, Israr UI, 1C \\
\hline Aksnes, Astrid, OA & Hawley, R. D., 10 \\
\hline Amar-Lewis, Eliz, 1A & Hirvonen, Liisa M., IN \\
\hline Amil, Pablo, OS & Hjelme, Dag Roar, OA \\
\hline Andersson-Engels, Stefan, $1 \mathrm{~N}$ & Hortholary, Thomas, ОT \\
\hline Anfimov, Dmitriy R., 1J & Høvik, Jens, OA \\
\hline Arivazhagan, A., 02 & Izosimova, Anna V., OV \\
\hline Ataman, Çaǵlar, Ol & Jernelv, Ine L., OA \\
\hline Azimani, Hicham, OF & Kamensky, Vladislav A., OV \\
\hline Balmages, llya, 1D & Kawahara, Yoshinobu, $1 \mathrm{C}$ \\
\hline Baver, Christoph, 0 l & Kenzhebayeva, Yuliya, IT \\
\hline Baum, O. I, OG & Kil'deeva, Nataliya, IR \\
\hline Bayhaqi, Yakub A., OK & Kiseleva, Elena B., 10 \\
\hline Bliznuks, Dmitrijs, 1D & Klämpfl, Florian, IF \\
\hline Blümel, Reinhold, OC & Kohler, Achim, OC \\
\hline Bradu, Adrian, $1 \mathrm{E}$ & Kolesova, Ekaterina P., 1A \\
\hline Brandsrud, Maren Anna, OC & Kost, Joseph, 1A \\
\hline Bratchenko, Ivan A., 06 & Kozlov, Sergey V., 06 \\
\hline Canbaz, Ferda, OK & Kretschmer, Simon, ol \\
\hline Caponi, Silvia, 08 & Krumina, Gunta, IW \\
\hline Capozzoli, Laura, 08 & Kumar, N. S., 04 \\
\hline Carminati, Rémi, OM & Kumar, Srividya, 02 \\
\hline Carrion, Claire, OT & Kuznetsov, Sergey S., OE, 10 \\
\hline Cattin, Philippe C., OK & Le galudec, Joël, OB \\
\hline Chen, Zih-Ting, $1 \mathrm{P}$ & Lefort, Claire, OT \\
\hline Chiang, Huihua Kenny, IP & Levecq, Olivier, OF \\
\hline Cicchi, Riccardo, 08 & Li, Lichuan, 09 \\
\hline Cižmár, Tomáš, 07 & Liduma, Sanita, IW \\
\hline Dadadzhanova, Antonina I., IA & Liepins, Janis, ID \\
\hline Dobre, George, $1 \mathrm{E}$ & Lifenko, Roman, $1 \mathrm{R}$ \\
\hline Dubois, Arnaud, OF & Lihachev, Alexey, 1D \\
\hline Dudenkova, Varvara V., 10 & Lin, Wai Ching, OP \\
\hline Duma, Virgil-Florin, $1 \mathrm{E}$ & Lisovskaya, Mariya G., 06 \\
\hline Dupoy, Mathieu, OB & Luguzis, Artis, IW \\
\hline Elagin, Vadim V., 10 & Lukina, Maria M., OV \\
\hline Epp, Hendrik, IF & Magnussen, Eirik A., OC \\
\hline Erdelyi, Ralph-Alexandru, $1 \mathrm{E}$ & Marcoux, Pierre R., OB \\
\hline Fioretto, Daniele, 08 & Maslov, Vladimir G., IA \\
\hline Franke-Arnold, S., 10 & Masoller, Cristina, OS \\
\hline Fufurin, Igor L., $1 \mathrm{~J}$ & Matcher, Stephen J., 09, OP \\
\hline Gavrina, Alena I., OV & Mattana, Sara, 08 \\
\hline Gelikonov, Grigory V., 1H & Matveev, Lev A., $\mathrm{OE}, \mathrm{OG}, \mathrm{OH}, 1 \mathrm{H}$ \\
\hline Gladkova, Natalia D., $\mathrm{OE}, \mathrm{OH}, 10$ & Matveyev, Alexander L., $\mathrm{OE}, \mathrm{OG}, \mathrm{OH}, 1 \mathrm{H}$ \\
\hline Goldbart, Riki, 1A & Mercatelli, Raffaella, 08 \\
\hline Golyak, Igor S., $1 \mathrm{~J}$ & Metelin, Vladislav, IR \\
\hline González-Cerdas, Gerardo E., Ol & Meyer, Tobias, 07 \\
\hline Grigorenko, Konstantin, $1 \mathrm{~T}$ & Moiseev, Alexander A., 1H, 10 \\
\hline Gubarkova, Ekaterina $\vee ., \mathrm{OE}, \mathrm{OH}, 1 \mathrm{O}$ & Moryatov, Alexander A., 06 \\
\hline Hamidi, Arsham, OK & Moskalensky, Alexander E., OU \\
\hline
\end{tabular}


Mukherjee, R., 04

Myakinin, Oleg O., 06

Navarini, Alexander, OK

Nomerotski, Andrei, $1 \mathrm{~N}$

Offer, R., 10

Ogien, Jonas, OF

Omelchenko, A. I., OG

Orlova, Anna O., IA

Papkovsky, Dmitri, IN

Pavone, Francesco S., 08

Perrot, Jean-Luc, OF

Pikálek, Tomáš, 07

Pini, Roberto, 08

Plekhanov, Anton A., OE

Podoleanu, Adrian Gh., $1 \mathrm{E}$

Polishchuk, Anton, IT

Popov, Evgeny, IT

Popp, Jürgen, 07

Radwell, N., 10

Ratto, Fulvio, 08

Rebuffel, Véronique, OB

Revin, Dmitry G., OP

Rossi, Francesca, 08

Sandic, Dragan, 01

Santhosh, Vani, 02

Savchenko, E. A., $1 U$

Schmidt, Michael, IF

Seim, Eivind, OC

Sen, Rajannya, iN

Šerý, Mojmír, 07

Serzhantov, Kirill A., 06

Shikhina, Nina, IR

Shirmanova, Marina $\vee$., OV

Sil, S., 04

Siret, David, OF

Sirotkina, Marina A., OE, 10

Sobol, Emil N., OG

Solheim, Johanne, OC

Somasundaram, Kumaravel, 02

Sovetsky, Alexander A., $\mathrm{OE}, \mathrm{OG}, \mathrm{OH}, 1 \mathrm{H}$

Späth, Moritz, IF

Spiryova, Darya V., OU

Stein, Patrick, Ol

Svihra, Peter, $1 \mathrm{~N}$

Tabalina, Anastasiya S., $1 \mathrm{~J}$

Temnov, Andrey, 1R

Trägårdh, Johanna, 07

Traitel, Tamar, 1A

Tricoli, Ugo, OM

Umapathy, Siva, 02, 04

Vasilenko, Irina, $1 \mathrm{R}$

Velichko, E. N., IU

Visvanathan, Abhirami, 02

Vitkin, Vladimir, IT

Vorob'ev, Alexei Yu., OU

Vorontsov, Alexey Yu., 10

Vorontsov, Dmitry A., 10

Wang, Chih-Hao, IP

Yuzhakov, A. V., OG

Yuzhakova, Diana V., OV
Zagaynova, Elena $\mathrm{V} ., \mathrm{OE}, \mathrm{OV}, 10$

Zaitsev, Vladimir Y., OE, OG, OH, 1H

Zakharov, Valery P., 06

Zam, Azhar, OK

Zappe, Hans, 0 I

Zhdanov, Alexander, $1 \mathrm{~N}$

Zolins, Stivens, 1D

Zykov, Alexey A., $1 \mathrm{H}$ 


\title{
Conference Committee
}

\author{
Symposium Chairs
}

Francis Berghmans, Vrije Universiteit Brussel (Belgium)

Thierry Georges, Oxxius SA (France)

Paul C. Montgomery, Université de Strasbourg (France)

Lluis Torner, ICFO Barcelona (Spain)

Conference Chairs

Jürgen Popp, Leibniz-Institut für Photonische Technologien e.V. (Germany)

Csilla Gergely, Laboratoire Charles Coulomb (France)

Conference Programme Committee

Peter E. Andersen, Technical University of Denmark (Denmark)

James M. Brewer, University of Glasgow (United Kingdom)

Arthur E. T. Chiou, National Yang-Ming University (Taiwan)

Jürgen W. Czarske, TU Dresden (Germany)

Johannes F. de Boer, Vrije Universiteit Amsterdam (Netherlands)

Kishan Dholakia, University of St. Andrews (United Kingdom)

Dror Fixler, Bar-llan University (Israel)

Sylvain Gioux, Université de Strasbourg (France)

Kirill V. Larin, University of Houston (United States)

Qingming Luo, Hainan University (China)

Thomas G. Mayerhöfer, Leibniz-Institut für Photonische Technologien e.V. (Germany)

Vasilis Ntziachristos, Helmholtz Zentrum München GmbH (Germany)

David D. Sampson, The University of Western Australia (Australia)

Ernst H. K. Stelzer, Goethe-Universität Frankfurt am Main (Germany)

Hugo Thienpont, Vrije Universiteit Brussel (Belgium)

Siva Umapathy, Indian Institute of Science (India)

I. Alex Vitkin, Ontario Cancer Institute (Canada)

Gert von Bally, Center for Biomedical Optics and Photonics

(Germany)

Brian C. Wilson, Princess Margaret Hospital (Canada)

Session Chairs

1 Raman Spectroscopy Imaging I

Jürgen Popp, Leibniz-Institut für Photonische Technologien e.V.

(Germany) 
2 Raman Spectroscopy Imaging II

Siva Umapathy, Indian Institute of Science, Bengaluru (India)

3 IR Spectroscopy and Imaging

Thomas G. Mayerhöfer, Leibniz-Institut für Photonische Technologien e.V. (Germany)

4 Optical Coherence Tomography I

Zhenyang Ding, Tianjin University (China)

5 Optical Coherence Tomography II

Vladimir Y. Zaitsev, Institute of Applied Physics of the RAS

(Russian Federation)

6 Optical Coherence Tomography III

Vishal Srivastava, Thapar Institute of Engineering and Technology (India)

$7 \quad$ Advanced Spectroscopy and Imaging I

Csilla Gergely, Laboratoire Charles Coulomb (France)

8 Advanced Spectroscopy and Imaging II

Cristina Masoller, Universitat Politècnica de Catalunya (Spain)

9 Advanced Spectroscopy and Imaging III

Claire Lefort, XLIM (France)

10 Advanced Spectroscopy and Imaging IV

Lei Xi, Southern University of Science and Technology of China (China) 